01

\title{
Влияние релятивистских взаимодействий на спектральные характеристики основного состояния монооксида углерода
}

\author{
() Е.А. Коновалова ${ }^{1}$, Ю.А. Демидов ${ }^{1,2}$, А.В. Столяров ${ }^{3}$ \\ ${ }^{1}$ Петербургский институт ядерной физики им. Б.П. Константинова Национального исследовательского центра \\ „Курчатовский институт“, \\ 188300 Гатчина, Россия \\ ${ }^{2}$ Санкт-Петербургский государственный электротехнический университет „ЛЭТИ“, \\ 197376 Санкт-Петербург, Россия \\ ${ }^{3}$ Химический фракультет, Московский государственный университет имени М.В. Ломоносова, \\ 119991 Москва, Россия \\ e-mail: lenaakonovalova@gmail.com
}

Поступила в редакцию 05.07.2018 г.

\begin{abstract}
Высокоточными неэмпирическими методами современной квантовой химии исследовано влияние слабых релятивистских взаимодействий на потенциальную энергию и собственный дипольный момент основного электронного состояния $X^{1} \Sigma^{+}$молекулы СО. Релятивистская энергия рассчитана тремя альтернативными методами: в первом порядке теории возмущений, используя оператор Коуэна-Гриффина, содержащий сумму масс-скоростной и дарвинской поправки, в рамках приближенного скалярного гамильтониана ДугласаКролла-Гесса, а также наиболее строгого „четырехкомпонентного“ релятивистского гамильтониана ДиракаКулона-Гаунта. Релятивистская поправка, полученная различными методами, согласуется в пределах нескольких процентов и составляет $\sim 55-60 \mathrm{~cm}^{-1}$ в районе равновесного межъядерного расстояния $R_{e}=1.128 \AA$. Учет релятивистской поправки приводит к уменьшению равновесной длины связи приблизительно на $0.0002 \AA$. Величина лэмбовского сдвига, оценная полуэмпирическим масштабированием одноэлектронного дарвинского терма, не превышает нескольких обратных сантиметров вблизи $R_{e}$. Релятивистская коррекция к функции дипольного момента составляет от - $0.001 \mathrm{D}$ до $+0.003 \mathrm{D}$, что не превышает $1 \%$ от нерялитивисткой составляющей дипольного момента.
\end{abstract}

DOI: $10.21883 /$ OS.2018.10.46693.98-18

\section{Введение}

Еще в 1937 г. Поль Дирак сформулировал гипотезу больших чисел [1], допускающую изменение фундаментальных физических постоянных с течением времени. Современные теории фундаментальных взаимодействий (великого объединения, суперструн и др.), пытающиеся объединить все известные силы в природе, также предполагают возможное изменение значений фундаментальных физических констант на космологическом масштабе времени [2]. Возможные вариации фундаментальных физических констант могут быть обнаружены во множестве физических явлений, но рекордная точность, которая может быть достигнута при определении частот и длин волн спектральных линий в атомах и молекулах, делает спектроскопию наиболее реалистичным инструментом для проверки данной гипотезы [3].

Поиск вариаций фундаментальных постоянных основан на сравнении линейчатых спектров атомов или молекул, полученных от космических объектов ранней Вселенной (квазаров с большим красным смещением $z$ ) $\lambda^{\mathrm{Q}}$, и зарегистрированных в наши дни в лабораторных условиях на Земле $\lambda^{\text {lab }}$. Изучаются вариации во времени безразмерных физических констант, таких как постоянная тонкой структуры $\alpha=\frac{e^{2}}{\hbar c}(e-$ заряд электрона, $c-$ скорость света, $\hbar-$ постоянная Планка) и отношение масс покоя электрона и протона $\mu=\frac{m_{e}}{m_{p}}$, для которых лабораторные спектральные измерения и астрономические наблюдения на больших красных смещениях представляют возможность непосредственного сопоставления

$$
\frac{\lambda_{i j}^{\mathrm{Q}}}{\lambda_{i j}^{\mathrm{lab}}}=(1+z)\left[1+K_{i j}^{\alpha} \frac{\Delta \alpha}{\alpha}+K_{i j}^{\mu} \frac{\Delta \mu}{\mu}\right],
$$

где коэффициенты чувствительности $K_{i j}^{x}$ отдельных линий $\lambda_{i j}=E_{i}-E_{j}$ определяются частными производными полных энергий атомных или молекулярных состояний $E_{i}$ и $E_{j}$ к варьируемому параметру $x \in \alpha ; \mu$

$$
K_{i j}^{x}=\frac{\partial \ln \left(\lambda_{i j}\right)}{\partial \ln (x)}=\frac{x}{E_{j}-E_{i}} \frac{\partial\left(E_{i}-E_{j}\right)}{\partial x} .
$$

Согласно диагональной теореме Гельмана-Феймана, необходимые производные энергий могут быть вычислены как среднее значение соответствующих производных полного гамильтониана $(H)$ :

$$
\frac{\partial E_{i}}{\partial x}=\left\langle\Psi_{i}\left|\frac{\partial H}{\partial x}\right| \Psi_{i}\right\rangle
$$

Зависимость длины волны и интенсивности спектральных линий от $\alpha$ возникает вследствие учета релятивистских $\left(H^{\text {rel }} \sim \alpha^{2} Z^{2}\right)$ и квантово-электродинамических $\left(H^{\mathrm{QED}} \sim \alpha^{3} Z[4]\right)$ поправок к кулоновской энергии связи 
электронов с ядром и энергии межэлектронного взаимодействия:

$$
\alpha \frac{\partial H}{\partial \alpha} \approx 2 H^{\mathrm{rel}}+3 H^{\mathrm{QED}} .
$$

Обе поправки пропорциональны зарядовому числу атома $Z$, поэтому для поиска вариации параметра $\alpha$ наиболее подходят тяжелые атомы и/или содержащие их молекулы. Для астрофизически значимых элементов, которые ограничены величинами $Z<30$, вклад релятивистских поправок в энергию электронного перехода обычно составляет несколько процентов. Очевидно, что зависимость электронного (ровибронного) спектра самой распространенной молекулы во Вселенной (молекулярного водорода) от $\alpha$ пренебрежимо мала изза минимального значения $Z=1$. Напротив, постоянная $\mu$ влияет только на изотопические сдвиги атомных спектров, вклады которых в энергии переходов крайне малы, тогда как наличие дополнительных (по сравнению с атомами) колебательных и вращательных степеней свободы у молекул делает зависимость именно молекулярных спектров от $\mu$ значительно более заметной, чем в атомах. По этим причинам космологический поиск вариаций $\alpha$ и $\mu$ обычно осуществляется раздельно с использованием атомных и молекулярных спектров соответственно [5-8]. Предполагая, что $\alpha$ и $\mu$ линейно зависят от времени, на данный момент получены следующие ограничения: $\dot{\alpha} / \alpha=(1.6 \pm 2.3) \times 10^{-17} /$ год [9] и $\dot{\mu} / \mu=(1.6 \pm 1.7) \times 10^{-15} /$ год [10].

Астрономические спектры второй по распространенности двухатомной молекулы во Вселенной - монооксида углерода $(\mathrm{CO})$ - неоднократно наблюдались на рекордно больших красных смещениях [11-13], что позволяет использовать ее для поиска предполагаемых вариаций фундаментальных постоянных на космологическом масштабе времени. Помимо ровибронных спектров, наличие собственного дипольного момента $d$ у молекулы СО допускает наличие у нее чисто вращательных и колебательно-вращательных переходов, чувствительность которых к вариации $\alpha$ и $\mu$, как следует из (2), должна быть существенно выше, чем у электронных. В собственный дипольный момент молекулы $\mathrm{CO} d(R)$ вносят вклад заряды обоих ядер $\left(Z_{\mathrm{C}}\right.$ и $\left.Z_{\mathrm{O}}\right)$, удаленные друг от друга на межъядерное расстояние $R$, а также неравномерное по $R$ распределение электронной плотности. Очевидно, что только электронная составляющая дипольного момента может зависеть от $\alpha$.

Недавно совместные ограничения на вариацию $\alpha$ и $\mu$ были получены из сравнения отношения сверхтонкого перехода атомарного углерода к вращательному переходу молекулы $\mathrm{CO}\left(\mathrm{C}_{\mathrm{fs}} / \mathrm{CO}_{\mathrm{rot}}\right)[14]$, однако при этом использовалось предположение, что вращательные уровни молекулы СО не зависят от $\alpha$.

В настоящее время использование спектров сверхвысокого разрешения ультрахолодных двухатомных молекул позволяет искать вариации фундаментальных постоянных во времени исключительно в лабораторных условиях, для чего используются квазивырожденные уровни разной симметрии $[15,16]$ или вырождение между колебательными уровнями и уровнями тонкой структуры [17]. Так, для поиска вариации $\mu$ был исследован двухфотонный переход между квазивырождеными вращательными уровнями в тонкой структуре возбужденных уровней метастабильного состояния $a^{3} П$ монооксида углерода [18].

В настоящей работе высокоточными методами неэмпирической квантовой химии изучено влияние относительно слабых релятивистских взаимодействий на потенциальную энергию и собственный дипольный момент основного электронного состояния $X^{1} \Sigma^{+}$молекулы $\mathrm{CO}$ с целью оценки чувствительности вращательных и колебательно-вращательных спектров различных изотопологов молекулы СО к предполагаемой вариации постоянной тонкой структуры.

\section{Детали релятивистского расчета электронной структуры}

Основной методикой решения многоэлектронной задачи при неэмпирическом расчете корреляционной энергии в настоящей работе послужил одноконфигурационный вариант итерационного метода связанных кластеров уровня CCSD и $\operatorname{CCSD}(\mathrm{T})$, который реализован в программном комплексе DIRAC15 [19] и специально ориентирован на учет релятивистских эффектов высокого уровня. Все 14 электронов молекулы монооксида углерода явно учитывались при расчете электронной корреляции. На этапе построения конфигурационного пространства применялся „четырехкомпонентный“ релятивистский гамильтониан Дирака-Кулона-Гаунта (DCG), однако, при учете электронной корреляции вклад от взаимодействия Гаунта не учитывался. В расчетах использовались корреляционно ориентированные базисные наборы aug-cс-pVnZ с кординальным числом $n=3$ и 4 [20]. Экстраполяция корреляционной энергии $E_{\mathrm{CC}}^{(\mathrm{n})}$ к бесконечному базисному набору (CBS) $E_{\mathrm{CC}}^{(\mathrm{CBS})}$ осуществлялась с помощью двухточечной эмпирической формулы:

$$
E_{\mathrm{CC}}^{(n)}=E_{\mathrm{CC}}^{(C B S)}+\frac{A}{(n+p)^{3}},
$$

где параметр $p \equiv 0$.

Собственный дипольный момент $d(R)$ вычислялся методом конечного электрического поля (CCSD-FF и $\operatorname{CCSD}(\mathrm{T})-\mathrm{FF})$ и также экстраполировался к бесконечному базису по формуле (5). Для оценки отклика электронной энергии на гипотетическую вариацию постоянной тонкой структуры $\alpha$ релятивистские расчеты выполнялись для трех значений параметра $\alpha=\frac{1}{137.0359922594}$, $\alpha_{1}=\sqrt{\frac{7}{8}} \alpha$ и $\alpha_{2}=\sqrt{\frac{9}{8}} \alpha$, как было предложено в работе [21]. Релятивистский вклад в полную энергию квадратично зависит от $\alpha$, что позволило выполнить интерполяцию результатов расчетов для различных значений $\alpha$ к нерелятивистскому пределу и выделить релятивистскую 
поправку $U^{\mathrm{rel}}(R)$ к энергии основного электронного состояния как функцию от межъядерного расстояния.

Для построения кривой потенциальной энергии $U(R)$ и функции дипольного момента $d(R)$ расчеты электронной энергии молекулы СО одноконфигурационными методами $\operatorname{CCSD}$ и $\operatorname{CCSD}(\mathrm{T})$ осуществлялись вблизи равновесного межъядерного расстояния $R_{e} \approx 1.128 \AA$ в относительно узком интервале $R \in[0.8,1.7] \AA$. Ограничение по $R$ вызвано тем, что используемые одноконфигурационные варианты метода связанных кластеров при больших межъядерных расстояниях начинают расходиться.

Для контроля реалистичности выполненных одноконфигурационных расчетов и возможного расширения диапазона исследуемых межъядерных расстояний электронная энергия основного состояния молекулы СО дополнительно рассчитывалась многоконфигурационным методом усредненных связанных пар [22] (MR$\mathrm{ACPF}$ ) с учетом суперпозиционной ошибки используемого базисного набора (BSSE) [23]. Величина скалярной релятивистской поправки была оценена двумя альтернативными методами: во-первых, по теории возмущений, используя оператор Коуэна-Гриффина, содержащий масс-скоростную и дарвинскую поправки, а, во-вторых, в рамках квазирелятивистского одноэлектронного гамильтониана Дугласа-Кролла-Гесса (DKH) [24]. В первом случае для нерелятивистского расчета энергий и дипольного момента использовался базисный набор nZaPa-NR с $n=4-7$ [25], специально оптимизированный для экстраполяции к бесконечному базису. Во втором случае мы использовали контрактированный базисный набор Дугласа-Крола [24] aug-cc-pwCV5Z-DK, адаптированный для проведения скалярных релятивистских расчетов с гамильтонианом DKH. Экстраполяция к бесконечному базисному набору нерелятивистских энергий, полученных методом MR-ACPF в интервале $R \in[0.6,2.6] \AA$ с использованием базисов $(n=4-6) \mathrm{ZaPa}-N R$, осуществлялась с помощью трехточечой экспоненциальной формулы

$$
E_{\mathrm{ACPF}}^{(\mathrm{n})}=E_{\mathrm{ACPF}}^{(\mathrm{CBS})}+A e^{-\beta n},
$$

a также с помощью степенной зависимости (5) при фиксированном параметре $p \equiv 0.5$ для базисов $(n=6,7) \mathrm{ZaPa}-\mathrm{NR}$.

Для оценки средних значений операторов КоуэнаГриффина и дипольного момента использовались нерелятивистские электронные волновые функции, полученные внутренне контрактированным многоссылочным методом конфигурационного взаимодействия (MR-CI) [26]. Расчеты методами MR-ACPF и MR-CI были выполнены с помощью программного комплекса MOLPRO [27], при этом явно коррелировались все 14 электронов молекулы СО, распределенных в полном активном пространстве оптимизированных молекулярных орбиталей [28].

Квантово-электродинамическая поправка к полной энергии легких атомов в основном определяется величиной лэмбовского сдвига, строгий квантово-механический

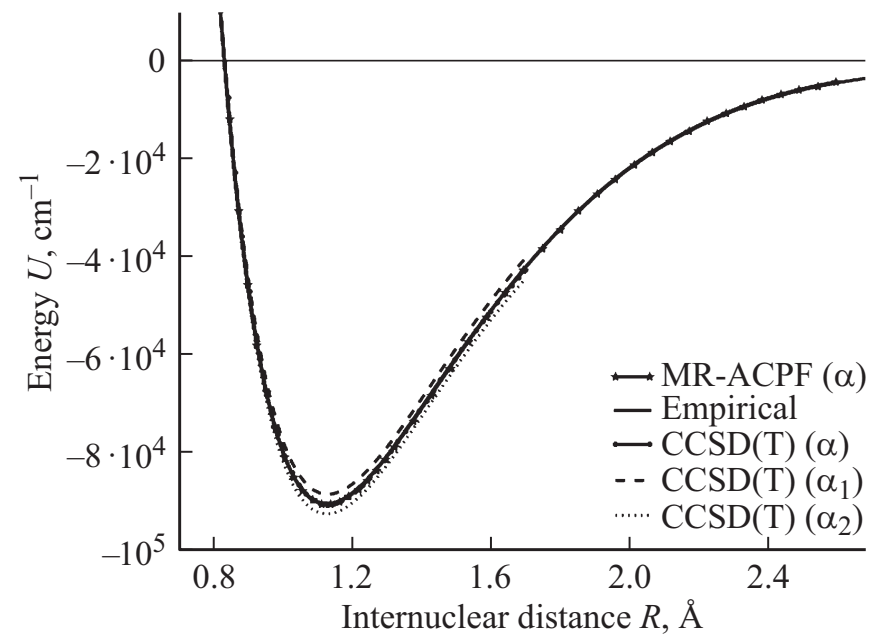

Рис. 1. Релятивистские потенциалы межатомного взаимодействия для основного электронного состояния $X^{1} \Sigma^{+}$молекулы $\mathrm{CO}$, рассчитанные в рамках MR-ACPF и $\operatorname{CCSD}(\mathrm{T})$ методов учета электронной корреляции для различных значений постоянной тонкой структуры $\alpha$. Эмпирический потенциал заимствован из работы [30].

расчет которого даже для атомов является нетривиальной задачей. Поэтому для оценки величины лэмбовского сдвига в относительно легких молекулах таких, например, как СО обычно используется приближенная полуэмпирическая схема [29], основанная на пропорциональности энергии лэмбовского сдвига $E^{\mathrm{Lamb}}$ одноэлектронной дарвинской поправке $E^{\text {Darwin. }}$

$$
\frac{E^{\text {textLamb }}}{E^{\text {Darwin }}} \approx \frac{2 \alpha}{\pi} F(\alpha Z)-\frac{8 \alpha}{15 \pi},
$$

где функция $F(\alpha Z)$ табулирована для валентных электронов атомов с зарядом $Z=1$ до $Z=54$ в табл. 1 работы [29].

\section{Результаты и обсуждение}

Как нерелятивистские, так и релятивистские потенциалы межатомного взаимодействия молекулы $\mathrm{CO}$, полученные в рамках альтернативных методов расчета, хорошо согласуются друг с другом, а также с высокоточными эмпирическими данными (рис. 1). Согласно результатам, представленным в табл. 1 и на рис. 2, релятивистский потенциал и равновесные молекулярные константы (длина связи и энергия диссоциации) совпадают с их эмпирическими аналогами с относительной погрешностью $0.01-0.03 \%$. В нерелятивистском приближении равновесная длина связи $\mathrm{C}-\mathrm{O}$ увеличивается на $2 \times 10^{-4} \AA$. С увеличением постоянной тонкой структуры $\alpha$ возрастает релятивистское сжатие оболочек с низшим значением полного момента $(j=1 / 2)$, что приводит к постепенному уменьшению равновесной длины связи $\mathrm{C}-\mathrm{O}$. 


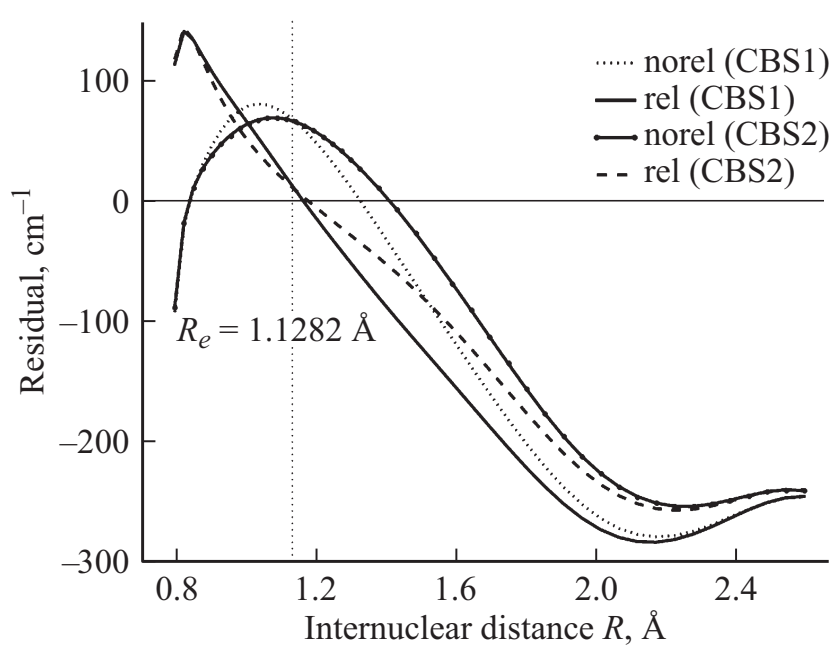

Рис. 2. Разность между высоточным эмпирическим потенциалом состояния $X^{1} \Sigma^{+}$молекулы СО [30] и его нерелятивистскими и релятивистскими аналогами, полученными при использовании двух различных вариантов экстраполяции MR$\mathrm{ACPF}$ энергий к бесконечному базису: CBS1 - (5) и CBS2 (6).

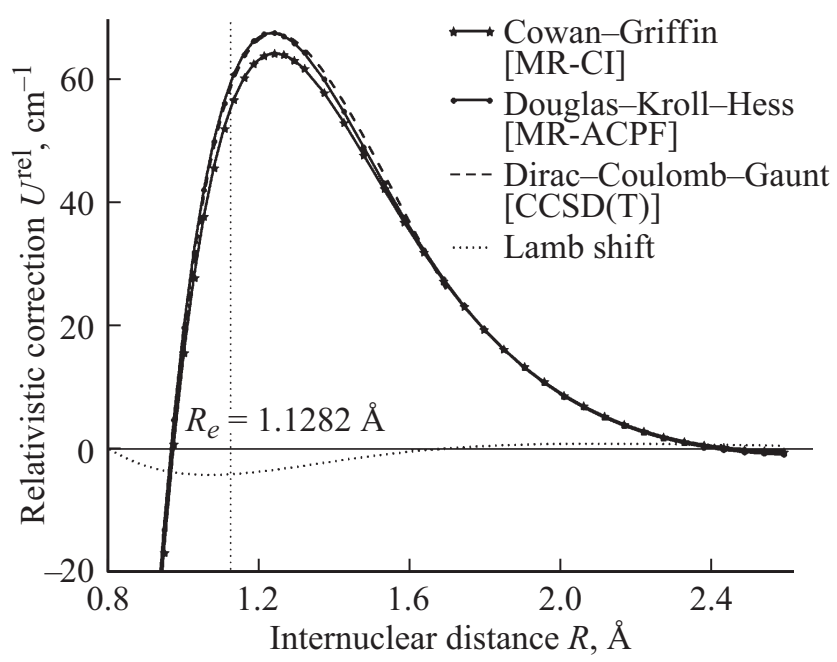

Рис. 3. Релятивистские поправки $U^{\mathrm{rel}}(R)$ к потенциальной энергии основного состояния молекулы СО как функции межъядерного расстояния $R$. Скалярная релятивистская энергия была рассчитана тремя альтернативными методами: (1) в 1ом порядке теории возмущений, используя оператор КоуэнаГриффина; (2) в рамках скалярного гамильтониана ДугласаКролла-Гесса и (3) „четырехкомпонентного“релятивистского гамильтониана Дирака-Кулона-Гаунта. Приближенная функция лэмбовского сдвига оценена полуэмпирически путем масштабированием одноэлектронной дарвинской поправки (7).

Зависимость релятивистской поправки к потенциальной энергии основного состояния молекулы $\mathrm{CO} U^{\mathrm{rel}}(R)$ от межъядерного расстояния $R$ представлена на рис. 3 . Эта зависимость может быть аппроксимированана трехпараметрической функцией Морзе:

$$
U^{\mathrm{rel}}(R)=U_{e}\left[2 e^{-a\left(R-R_{m}\right)}-e^{-2 a\left(R-R_{m}\right)}\right] .
$$

Таблица 1. Скорость сходимости равновесных молекулярных параметров электронной структуры (межъядерного расстояния $R_{e}$ и энергии диссоциации $D_{e}$ ) основного состояния $X^{1} \Sigma^{+}$молекулы СО в зависимости от полноты используемого базисного набора и уровня учета электронной корреляции. Соответствующие эмпирические данные взяты из работы [30]

\begin{tabular}{l|c|c}
\hline & $R_{e}, \AA$ & $D_{e}, \mathrm{~cm}^{-1}$ \\
\hline \multicolumn{3}{c}{ Nonrel-ACPF/nZaPa-NR } \\
\hline$n=4$ & 1.1312 & 89570 \\
$n=5$ & 1.1297 & 90248 \\
$n=6$ & 1.1287 & 90536 \\
$n=7$ & 1.1287 & 90603 \\
\hline \multicolumn{3}{c}{ Nonrel-ACPF } \\
\hline CBS1 & 1.1282 & 90740 \\
CBS2 & 1.1281 & 90740 \\
\hline \multicolumn{3}{c}{ Rel-ACPF } \\
\hline CBS1 & 1.1280 & 90685 \\
\hline CCSD/CBS1 & 1.1216 & 90678 \\
CCSD(T)/CBS1 & 1.1283 & \\
\hline Empirical & 1.1282 &
\end{tabular}

Функция $U^{\mathrm{rel}}(R) \quad$ достигает максимума $U_{e}=$ $=65 \pm 2 \mathrm{~cm}^{-1}$ при межьядерных расстояниях $R_{m}=$ $=1.24 \pm 0.02 \AA$, слегка превышающих равновесное расстояние $R_{e}$. Вклад взаимодействия Гаунта в $U^{\mathrm{rel}}(R)$ не превышает $1 \mathrm{~cm}^{-1}$. Скалярная релятивистская поправка, оцененная альтернативными методами, согласуется в пределах нескольких процентов и составляет $\sim 55-60 \mathrm{~cm}^{-1}$ в районе равновесного межьядерного расстояния. Величина лэмбовского сдвига, оценная полуэмпирическим масштабированием одноэлектронного дарвинского терма, составлет $3-4 \mathrm{~cm}^{-1}$ вблизи $R_{e}$.

Рассчитанные величины дипольного момента $d$ для различных значений $R$ представлены на рис. 4. Как и следовало ожидать, электронный вклад в дипольный момент молекулы СО существенно зависит от уровня учета электронной корреляции и степени полноты используемого базисного набора. Так величина дипольного момента, относящаяся к равновесному расстоянию, лишь в рамках методов $\mathrm{CCSD}(\mathrm{T}) / \mathrm{CBS}$ и MR-ACPF/CBS согласуется с экспериментальными данными [31]. Влияние релятивистских взаимодействий на функцию дипольного момента, рассчитанную методом конечного поля и как среднее значение оператора дипольного момента, составляет 0.001-0.003 D (рис. 5), что, однако, не превышает $1 \%$ от нерялитивисткой составляющей абсолютной величины дипольного момента.

Вблизи положения равновесия дипольный момент молекулы СО почти линейная функция межьядерного расстояния $R$, поэтому при расчете отклика дипольного 


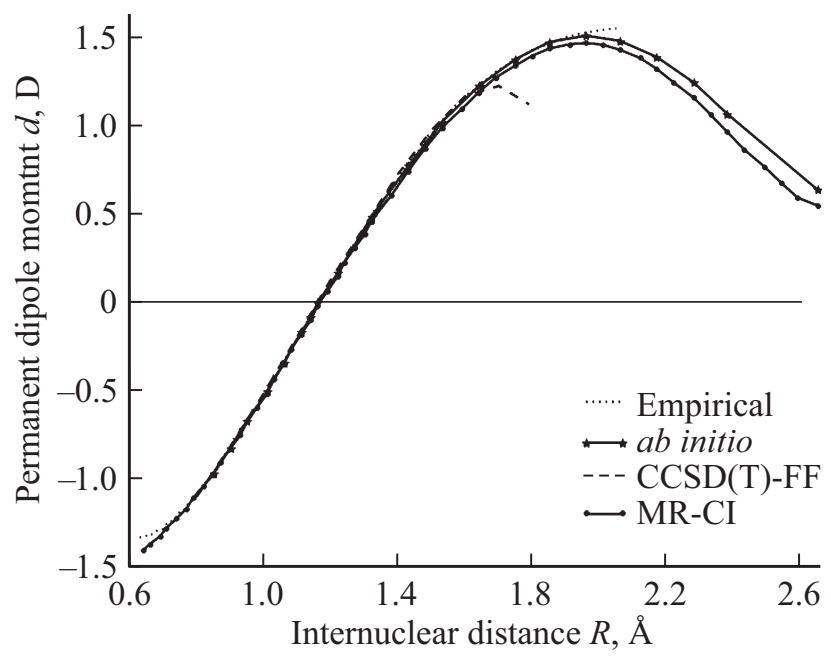

Рис. 4. Зависимость дипольного момента молекулы СО от межъядерного расстояния $R$, построенная по данным расчетов методами $\mathrm{CCSD}(\mathrm{T})-\mathrm{FF}$ и MR-ACPF в пределе бесконечного базисного набора. Направление дипольного момента ${ }^{-} \mathrm{CO}^{+}$. Эмпирическая функция дипольного момента взята из работы [31], а неэмпирические (ab initio) данные Лангоффа из [32].

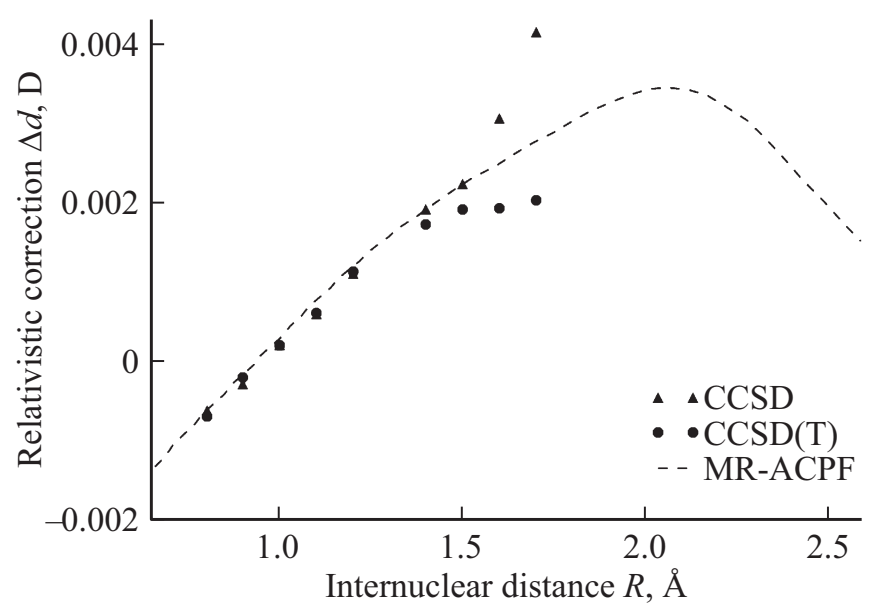

Рис. 5. Зависимость релятивистской поправки к дипольному моменту молекулы СО от межъядерного расстояния при $\alpha=\alpha_{0}$, построенная методами MR-ACPF, CCSD и $\operatorname{CCSD}(\mathrm{T})$ в пределе бесконечного базисного набора.

момента молекулы СО при равновесной длине связи на изменение постоянной тонкой структуры $\alpha$ необходимо также учитывать зависимость $R_{e}$ от $\alpha$ :

$\frac{\Delta d(\alpha, R)}{\Delta \alpha} \approx\left(\frac{\partial d(\alpha, R)}{\partial \alpha}\right)_{R=R_{e}}+\left(\frac{\partial d(\alpha, R)}{\partial R}\right)_{\alpha=\alpha_{0}}\left(\frac{\partial R_{e}}{\partial \alpha}\right)$.

Согласно численным результатам, полученным релятивистским методом CCSD(T)/CBS, оба слагаемых в выражении (9) дают сопоставимые вклады в значение отклика дипольного момента на изменение $\alpha: 0.1942 \mathrm{D}$ и 0.1458 D соответственно (см. также табл. 2).
Таблица 2. Отклик равновесных структурных параметров основного состояния молекулы $\mathrm{CO}$, рассчитанных релятивистским методом CCSD(T)/CBS-FF, на возможные вариации постоянной тонкой структуры $\alpha$

\begin{tabular}{l|c}
\hline$\partial R_{e} / \partial \alpha, \AA$ & -0.0473 \\
\hline$\frac{\partial D_{e} / \partial \alpha}{D_{e}}$ & 0.1734 \\
\hline$\partial d / \partial \alpha\left(R_{e}\right), \mathrm{D}$ & 0.1942
\end{tabular}

\section{Заключение}

Высокоточными неэмпирическими методами современной квантовой химии в ограниченном диапазоне межъядерных расстояний $R \in[0.6,2.6] \AA$ исследовано влияние скалярных релятивистских взаимодействий на межатомный потенциал и собственный дипольный момент состояния $X^{1} \Sigma^{+}$молекулы СО с целью последующей оценки коэффициентов чувствительности положения линий и интенсивностей колебательно-вращательных и чисто вращательных переходов к прогнозируемой вариации постоянной тонкой структуры $\alpha$ в космологическом масштабе времени. Результаты неэмпирических расчетов доступны в табличной форме от авторов по требованию.

Для дальнейшего повышения точности расчета и возможного расширения диапазона рассматриваемых межъядерных расстояний (прежде всего в сторону их увеличения) необходимо, по-видимому, использовать более трудоемкие многоконфигурационные варианты метода связанных кластеров (и/или конфигурационного взаимодействия), позволяющие адекватно учесть значительное возрастание многоконфигурационного характера электронной волновой функции основного состояния СО при средних и особенно больших $R$. Кроме того, для оценки чувствительности спектральных характеристик основного состояния монооксида углерода к возможной вариации отношения масс покоя электрона и протона $\mu$ потребуется дополнительный расчет адиабатической (диагональной) коррекции, а также масс-зависимых вкладов в эффективный колебательно-вращательный гамильтониан от регулярных гомогенных и гетерогенных неадиабатических взаимодействий с удаленными электронными состояниями.

Авторы благодарят М.Г. Козлова и Л.В. Скрипникова за плодотворные обсуждения. Работа поддержана Российским фондом фундаментальных исследований (проект РФФИ № 17-32-50022-мол-нр). Расчеты проведены с использованием оборудования центра коллективного пользования „Комплекс моделирования и обработки данных исследовательских установок мега-класса“ НИЦ „Курчатовский институт“. 


\section{Список литературы}

[1] Dirac P.A.M. // Nature. 1937. V. 139. N. 3512. P. 323.

[2] Uzan J.P. // Rev. Mod. Phys. 2003. V. 75. P. 403-455.

[3] Karshenboim S.G., Peik E. // EPJ Special Topics. 2008. V. 163. N. 1. P. 1-7.

[4] Собельман И.И. Введение в теорию атомных спектров. Наука, 1977.

[5] Molaro P., Centurion M., Whitmore J.B. et al. // Astron. Astrophys. 2013. V. 555. N. A68.

[6] Kozlov M.G., Levshakov S.A. // Annalen der Physik. 2013. V. 525. N 7. P. 452-471.

[7] Konovalova E.A., Kozlov M.G., Imanbaeva R.T. // Phys. Rev. A. 2014. V. 90. N. 4. P. 042512.

[8] Мешков В.В, Столяров А.В., Иванчик А.В., Варшалович Д.А. // Письма в ЖЭТФ. 2006. V. 83. N. 8. Р. 363-366.

[9] Rosenband T., Hume D.B., Schmidt P.O. et al. // Science. 2008. V. 319. N 5871. P. 1808-1812.

[10] Blatt S., Ludlow A.D., Campbell G.K. et al. // Phys. Rev. Lett. 2008. V. 100. N. 14. P. 140801.

[11] Barvainis R., Tacconi L., Antonucci R. et al. // Nature. 1994. V. 371. N 6498. P. 586.

[12] Papadopoulos P.P., Röttgering H.J.A., Van P.P., Werf D. et al. // Astrophys. J. 2000. V. 528. N 2. P. 626.

[13] Combes F., Rex M., Rawle T.D. et al. // A.\& A. 2012. V. 538. P. L4.

[14] Levshakov S.A., Combes F., Boone F. et al. // A.\&A. 2012. V. 540. P. L9.

[15] DeMille D., Sainis S., Sage J. et al. // Phys. Rev. Lett. 2008. V. 100. N 4. P. 043202.

[16] Zelevinsky T., Kotochigova S., Ye J. // Phys. Rev. Lett. 2008. V. 100. N 4. P. 043201.

[17] Flambaum V.V., Kozlov M.G. // Phys. Rev. Lett. 2007. V. 99. N 15. P. 150801.

[18] Bethlem H.L., Ubachs W. // Faraday discuss. 2009. V. 142. P. 25-36.

[19] Bast R., Saue T., Visscher L. et al. DIRAC, a relativistic ab initio electronic structure program, Release DIRAC15. 2015. http://www.diracprogram.org

[20] Dyall K.G. // Theor. Chem. Acc. 2006. V. 115. N 5. P. 441447.

[21] Dzuba V.A., Flambaum V.V., Webb J.K. // Phys. Rev. A. 1999. V. 59. N 1. P. 230.

[22] Werner H.J., Knowles P.J. // Theor. Chem. Acc. 1990. V. 78. N 3. P. $175-187$.

[23] Boys S.F., Bernardi F. // Mol. Phys. 1970. V. 19. N 4. P. $553-$ 566.

[24] Wolf A., Reiher M., Hess B.A. // J. Chem. Phys. 2002. V. 117. N 20. P. 9215-9226.

[25] Ranasinghe D.S., Petersson G.A. // J. Chem. Phys. 2013. V. 138. N 14. P. 144104.

[26] Werner H.J., Knowles P.J. // J. Chem. Phys. 1988. V. 89. P. 5803-5814.

[27] Werner H.-J., Knowles P. J., Knizia G. et al. MOLPRO, version 2010.1, a package of ab initio programs. 2010.

[28] Werner H.J., Knowles P.J. // J. Chem. Phys. 1985. V. 82. N 11. P. 5053-5063.

[29] Pyykkö P., Dyall K.G., Császár A.G. et al. // Phys. Rev. A. 2001. V. 63. P. 024502.

[30] Coxon J.A., Hajigeorgiou P.G. // J. Chem. Phys. 2004. V. 121. N 7. P. $2992-3008$
[31] Li G., Gordon I.E., Rothman L.S. et al. // Astrophys. J. Supplement. 2015. V. 216. N 1. P. 15.

[32] Langhoff S.R, Bauschlicher Jr. C.W. // J. Chem. Phys. 1995. V. 102. N 13. P. 5220-5225. 